\title{
Existence of Nontrivial Solutions for Some Second-Order Multipoint Boundary Value Problems
}

\author{
Hongyu Li $(\mathbb{D})$ and Junting Zhang \\ College of Mathematics and Systems Science, Shandong University of Science and Technology, Qingdao, Shandong, 266590, China \\ Correspondence should be addressed to Hongyu Li; sdlhy1978@163.com
}

Received 4 July 2018; Revised 7 November 2018; Accepted 25 November 2018; Published 11 December 2018

Academic Editor: Yong H. Wu

Copyright (C) 2018 Hongyu Li and Junting Zhang. This is an open access article distributed under the Creative Commons Attribution License, which permits unrestricted use, distribution, and reproduction in any medium, provided the original work is properly cited.

By using fixed point theorems with lattice structure, the existence of negative solution and sign-changing solution for some secondorder multipoint boundary value problems is obtained.

\section{Introduction}

In this paper, the following second-order ordinary differential equation will be considered:

$$
-x^{\prime \prime}(t)=\varphi(t, x(t)), \quad 0 \leq t \leq 1,
$$

subject to the multipoint boundary condition

$$
\begin{aligned}
& x(0)=0, \\
& x(1)=\sum_{i=1}^{m-2} \beta_{i} x\left(\alpha_{i}\right),
\end{aligned}
$$

where $\beta_{i}>0, i=1,2, \cdots, m-2 ; \sum_{i=1}^{m-2} \beta_{i}<1 ; 0<\alpha_{1}<\alpha_{2}<$ $\cdots<\alpha_{m-2}<1$, and $\sum_{i=1}^{m-2} \beta_{i} \alpha_{i}<1$.

The multipoint boundary value problems of ordinary differential equations arise in different areas of applied mathematics and physics. In 1992, Gupta studied nonlinear second-order three-point boundary value problems (see [1]). Since then, different types of nonlinear multipoint boundary value problems have been studied. Up to now, many great achievements about multipoint boundary value problems have been made. For example, many authors have investigated the existence of nontrivial solutions for nonlinear multipoint boundary value problems. Most of them have used upper and lower solution method, fixed point index theory, Guo-Krasnosel'skii fixed point theorem, bifurcation theory, fixed point theorems on cones, and so on (see [227] and references therein). For instance, in [2], the author considered the second-order multipoint boundary value problem

$$
\begin{aligned}
y^{\prime \prime}(t)+f(y) & =0, \quad 0 \leq t \leq 1, \\
y(0) & =0, \\
y(1) & =\sum_{i=1}^{m-2} \alpha_{i} y\left(\eta_{i}\right) .
\end{aligned}
$$

By using fixed point index and Leray-Schauder degree methods, the author showed existence of multiple sign-changing solutions for the boundary value problem (3). In [14], the authors have considered the following multipoint boundary value problem:

$$
\begin{aligned}
-(L \varphi)(t) & =\lambda f(t, \varphi(t)), \quad 0 \leq t \leq 1, \\
\varphi^{\prime}(0) & =0, \\
\varphi(1) & =\sum_{i=1}^{m-2} \beta_{i} \varphi\left(\eta_{i}\right) .
\end{aligned}
$$

The authors have used global bifurcation method to obtain the existence of positive solution of the boundary value problem (4).

In recent years, some authors combine the theory of lattice and the theory of topological degree, so they have obtained some fixed point theorems with lattice structure 
for nonlinear operators which are not assumed to be cone mappings (see [28-34]). At present, a few authors have used those fixed point theorems with lattice structure to study boundary value problems (see [6, 17, 28-37]). For example, in [35], by using fixed point theorems with lattice structure, the authors considered the existence of positive solution and sign-changing solution for integral boundary value problem under sublinear condition. In [37], the authors considered the existence of positive solution for fourth-order differential equation with fixed point theorems with lattice structure. In [6], the author considered the following second-order threepoint boundary value problem:

$$
\begin{aligned}
-u^{\prime \prime}(t) & =g(t, u(t)), \quad 0 \leq t \leq 1, \\
u(0) & =0, \\
u(1) & =\alpha u(\beta),
\end{aligned}
$$

where $g:[0,1] \times(-\infty,+\infty) \longrightarrow(-\infty,+\infty)$ is continuous, $0<\alpha<1,0<\beta<1$. The author used fixed point theorems with lattice structure to study the existence of sign-changing solutions for the boundary value problem (5) under the unilaterally asymptotically linear condition.

Motivated by $[6,17,28-37]$, we shall study the existence of nontrivial solutions for the boundary value problem (1), (2). In this paper, we assume that the nonlinear term satisfies superlinear conditions concerning the first eigenvalue corresponding to the relevant linear operator. The method we use is fixed point theorems with lattice structure. And we obtain the sufficient condition about the existence of negative solution and sign-changing solution for the boundary value problem (1), (2). The method is different from those of [2, 4]. And the main results are different from those of the work $[2,4]$. This paper is arranged as follows. In Section 2, we give some definitions and fixed point theorems with lattice structure. In Section 3, we shall give some lemmas and the main results about the existence of nontrivial solutions (including negative solution and sign-changing solution) for the boundary value problem (1), (2). Finally, in Section 4, some examples are given to illustrate our main results.

\section{Preliminaries}

Let $E$ be an ordered Banach space in which the partial ordering $\leq$ is induced by a cone $P \subset E$. $P$ is called normal if there exists a positive constant $N>0$ such that $\theta \leq u \leq v$ implies $\|u\| \leq N\|v\|$. $P$ is called solid if int $\mathrm{P} \neq \theta$, i.e., $P$ has nonempty interior. $P$ is called total if $E=\overline{P-P}$. If $P$ is solid, then $P$ is total. For the concepts and the properties about the cones, we refer to $[31,38,39]$.

We call $E$ a lattice under the partial ordering $\leq$, if $\sup \{u, v\}$ and $\inf \{u, v\}$ exist for arbitrary $u, v \in E$.

For $u \in E$, let

$$
\begin{aligned}
& u^{+}=\sup \{u, \theta\}, \\
& u^{-}=\sup \{-u, \theta\}
\end{aligned}
$$

$u^{+}$and $u^{-}$are called positive part and negative part of $u$, respectively. Taking $|u|=u^{+}+u^{-}$, then $|u| \in P$. For the definition and the properties of the lattice, we refer to [40].

For convenience, we use the following notations:

$$
\begin{aligned}
& u_{+}=u^{+}, \\
& u_{-}=-u^{-},
\end{aligned}
$$

and clearly

$$
\begin{aligned}
& u_{+} \in P, \\
& u_{-} \in(-P), \\
& u=u_{+}+u_{-} .
\end{aligned}
$$

Definition 1 (see [28-31]). Let $D \subset E$ and $F: D \longrightarrow E$ be a nonlinear operator. If there exists $u^{*} \in E$ such that

$$
F u=F u_{+}+F u_{-}+u^{*}, \quad \forall u \in D,
$$

then $F$ is said to be quasi-additive on lattice.

Let $B: E \longrightarrow E$ be a bounded linear operator. If $B(P) \subset P$, then the operator $B$ is called to be positive.

In this section, we assume that $E$ is a Banach space, $P$ is a total cone, the partial ordering $\leq$ in $E$ is induced by $P$, and $E$ is a lattice in the partial ordering $\leq$.

Let $B: E \longrightarrow E$ be a positive completely continuous linear operator; $B^{*}$ the conjugated operator of $B ; r(B)$ a spectral radius of $B$; and $P^{*}$ the conjugated cone of $P$. Since $P \subset E$ is a total cone, by Krein-Rutman theorem, we can infer that if $r(B) \neq 0$, then there exist $\bar{u} \in P \backslash\{\theta\}$ and $f^{*} \in P^{*} \backslash\{\theta\}$, such that

$$
\begin{aligned}
B \bar{u} & =r(B) \bar{u}, \\
B^{*} f^{*} & =r(B) f^{*} .
\end{aligned}
$$

For $\delta>0$. Let

$$
P\left(f^{*}, \delta\right)=\left\{u \in P \mid f^{*}(u) \geq \delta\|u\|\right\} .
$$

Then $P\left(f^{*}, \delta\right)$ is also a cone in $E$.

Definition 2 (see $[30,31,41]$ ). If there exist $\bar{u} \in P \backslash\{\theta\}, f^{*} \in$ $P^{*} \backslash\{\theta\}$, and $\delta>0$ such that (10) holds, and $B$ maps $P$ into $P\left(f^{*}, \delta\right)$, then the positive linear operator $B$ is said to satisfy $\mathrm{H}$ condition.

Let $P$ be a cone of a Banach space $E$. If $u \in(P \backslash\{\theta\})$ is a fixed point of $A$, then $u$ is said to be a positive fixed point of $A$. If $u \in((-P) \backslash\{\theta\})$ is a fixed point of operator $A$, then $u$ is said to be a positive fixed point of operator $A$. If $u \in(P \backslash\{\theta\})$ is a fixed point of operator $A$, then $u$ is said to be a negative fixed point of operator $A$. If $u \notin(P \cup(-P))$ is a fixed point of operator $A$, then $u$ is said to be a sign-changing fixed point of operator $A$.

In [30], Sun and Liu considered computation for the topological degree about superlinear operators which are not cone mappings and obtained the following results.

Lemma 3. Let the cone $P \subset E$ be solid, and $A: E \longrightarrow E$ be a completely continuous operator, and $A=B F$, where $B$ is 
a positive completely continuous linear operator satisfying $\mathrm{H}$ condition and $F$ is quasi-additive on lattice. Assume that

(i) there exist $c_{1}>r^{-1}(B)$ and $u_{1} \in P$ such that

$$
F u \geq c_{1} u-u_{1}, \quad \forall u \in P
$$

(ii) there exist $0<c_{2}<r^{-1}(B)$ and $u_{2} \in P$ such that

$$
F u \geq c_{2} u-u_{2}, \quad \forall u \in(-P) ;
$$

(iii) $A \theta=\theta$, the Fréchet derivative $A_{\theta}^{\prime}$ of $A$ at $\theta$ exists, and 1 is not an eigenvalue of $A_{\theta}^{\prime}$.

Then the operator $A$ has at least one nonzero fixed point.

In [31], Sun further obtained the following result about the existence of sign-changing fixed points for superlinear operators.

Lemma 4. Let the conditions in Lemma 3 hold, and $\beta$ denote the sum of the algebraic multiplicities for all eigenvalues of $A_{\theta}^{\prime}$ lying in $(1,+\infty)$. In addition, assume that

(iv) $\beta \neq 0, \beta$ is an even number;

(v) $A(P \backslash\{\theta\}) \subset \operatorname{int} P, A((-P) \backslash\{\theta\}) \subset \operatorname{int}(-P)$.

Then the operator $A$ has at least one negative fixed point and one sign-changing fixed point.

\section{Main Results}

For convenience, we list the following conditions. $[0,1]$.

$\left(\mathrm{C}_{1}\right) \varphi:[0,1] \times R^{1} \longrightarrow R^{1}$ is continuous, $\varphi(t, 0)=0, \forall t \in$

$\left(C_{2}\right)$ The sequence of positive solutions of the equation

$$
\sin \sqrt{y}=\sum_{i=1}^{m-2} \beta_{i} \sin \left(\alpha_{i} \sqrt{y}\right)
$$

is

$$
0<\lambda_{1}<\lambda_{2}<\cdots<\lambda_{n}<\lambda_{n+1}<\cdots .
$$

$\left(\mathrm{C}_{3}\right) \lim _{x \rightarrow 0}(\varphi(t, x) / x)=\eta$ uniformly on $t \in[0,1]$.

Let $X=C[0,1]$ with supremum norm $\|x\|=$ $\sup _{0 \leq t \leq 1}|x(t)|$. Set $P=\{x \in X \mid x(t) \geq 0, t \in[0,1]\}$, the $P$ is a solid cone in $X$. And under the partial order $\leq$ which is induced by $P, X$ is a lattice.

In the following, we define some operators $A, B$, and $\Phi$ :

$$
\begin{aligned}
& (A x)(t)=\int_{0}^{1} K(t, s) \varphi(s, x(s)) d s, \quad t \in[0,1], \\
& (B x)(t)=\int_{0}^{1} K(t, s) x(s) d s, \quad t \in[0,1], \\
& (\Phi x)(t)=\varphi(t, x(t)), \quad t \in[0,1],
\end{aligned}
$$

where

$$
\begin{gathered}
K(t, s)=g(t, s)+\frac{t \sum_{i=1}^{m-2} \beta_{i} g\left(\alpha_{i}, s\right)}{1-\sum_{i=1}^{m-2} \beta_{i} \alpha_{i}}, \\
g(t, s)= \begin{cases}t(1-s), & 0 \leq t \leq s \leq 1, \\
s(1-t), & 0 \leq s \leq t \leq 1 .\end{cases}
\end{gathered}
$$

Obviously, $A=B \Phi$, and the nontrivial fixed points of the operator $A$ are nontrivial solutions of the boundary value problem (1), (2) (see [3]).

Lemma 5 (see [2]). Let $\mu$ be a positive number, and the linear operator $B$ be defined by (17). Eigenvalues of the linear operator $\mu B$ are

$$
\frac{\mu}{\lambda_{1}}, \frac{\mu}{\lambda_{2}}, \cdots, \frac{\mu}{\lambda_{n}}, \cdots
$$

and algebraic multiplicity of $\mu / \lambda_{n}$ is equal to 1 , where $\lambda_{n}$ is defined by $\left(\mathrm{C}_{2}\right)$.

Lemma 6. The linear operator B satisfies $\mathrm{H}$ condition.

Proof. By $\left(\mathrm{C}_{2}\right)$, Lemma 5, and the definition of the spectral radius, we know that

$$
r(B)=\sup _{\lambda \in\left\{1 / \lambda_{n}, n=1,2, \cdots\right\}}|\lambda|=\frac{1}{\lambda_{1}}>0 .
$$

By (20), we have

$$
\begin{aligned}
& g\left(\alpha_{i}, s\right) \geq \alpha_{i}\left(1-\alpha_{i}\right) s(1-s), \quad \forall s \in[0,1] \\
& g(t, s) \leq s(1-s), \quad \forall t, s \in[0,1]
\end{aligned}
$$

By (19) and (23), we have

$$
\begin{aligned}
& K(t, s) \geq \frac{t \sum_{i=1}^{m-2} \beta_{i} g\left(\alpha_{i}, s\right)}{1-\sum_{i=1}^{m-2} \beta_{i} \alpha_{i}} \\
& \geq \frac{t \sum_{i=1}^{m-2} \beta_{i} \alpha_{i}\left(1-\alpha_{i}\right) s(1-s)}{1+\sum_{i=1}^{m-2} \beta_{i}\left(1-\alpha_{i}\right)}, \\
& \forall t, s \in[0,1] .
\end{aligned}
$$

From (24) and (25), we have

$$
K(t, s) \geq \frac{t \sum_{i=1}^{m-2} \beta_{i} \alpha_{i}\left(1-\alpha_{i}\right)}{1+\sum_{i=1}^{m-2} \beta_{i}\left(1-\alpha_{i}\right)} g(\tau, s),
$$

$$
\forall \tau, t, s \in[0,1] .
$$

By (19), we have

$$
\begin{array}{r}
K(t, s) \geq \frac{t \sum_{i=1}^{m-2} \beta_{i} g\left(\alpha_{i}, s\right)}{1-\sum_{i=1}^{m-2} \beta_{i} \alpha_{i}} \geq \frac{t \sum_{i=1}^{m-2} \beta_{i} \tau g\left(\alpha_{i}, s\right)}{1-\sum_{i=1}^{m-2} \beta_{i} \alpha_{i}} \\
\geq \frac{t \sum_{i=1}^{m-2} \beta_{i} \alpha_{i}\left(1-\alpha_{i}\right)}{1+\sum_{i=1}^{m-2} \beta_{i}\left(1-\alpha_{i}\right)} \frac{\sum_{i=1}^{m-2} \beta_{i} \tau g\left(\alpha_{i}, s\right)}{1-\sum_{i=1}^{m-2} \beta_{i} \alpha_{i}}, \\
\forall \tau, t, s \in[0,1] .
\end{array}
$$

Hence, by adding (26) to (27), we have

$$
\begin{aligned}
& 2 K(t, s) \geq t \frac{\sum_{i=1}^{m-2} \beta_{i} \alpha_{i}\left(1-\alpha_{i}\right)}{1+\sum_{i=1}^{m-2} \beta_{i}\left(1-\alpha_{i}\right)}(g(\tau, s) \\
& \left.+\frac{\sum_{i=1}^{m-2} \beta_{i} \tau g\left(\alpha_{i}, s\right)}{1-\sum_{i=1}^{m-2} \beta_{i} \alpha_{i}}\right)
\end{aligned}
$$


i.e.,

$$
K(t, s) \geq M t K(\tau, s), \quad \forall \tau, t, s \in[0,1],
$$

where $M=\sum_{i=1}^{m-2} \beta_{i} \alpha_{i}\left(1-\alpha_{i}\right) / 2\left(1+\sum_{i=1}^{m-2} \beta_{i}\left(1-\alpha_{i}\right)\right)$.

Let

$$
\left(B^{*} x\right)(t)=\int_{0}^{1} K^{*}(t, s) x(s) d s, \quad \forall t \in[0,1],
$$

where $K^{*}(t, s)=K(s, t)$. Obviously, $r\left(B^{*}\right)=r(B)=1 / \lambda_{1}>$ 0 . By Krein-Rutman theorem, there exist $x(t) \in P \backslash\{\theta\}$ and $x^{*}(t) \in P \backslash\{\theta\}$ such that

$$
\begin{aligned}
(B x)(t) & =r(B) x(t), \\
\left(B^{*} x^{*}\right)(t) & =r(B) x^{*}(t) .
\end{aligned}
$$

By (29) and (32), we obtain

$$
\begin{aligned}
x^{*}(s) & =r^{-1}(B)\left(B^{*} x^{*}\right)(s) \\
& =r^{-1}(B) \int_{0}^{1} K^{*}(s, t) x^{*}(t) d t \\
& =r^{-1}(B) \int_{0}^{1} K(t, s) x^{*}(t) d t \\
& \geq M r^{-1}(B) \int_{0}^{1} t K(\tau, s) x^{*}(t) d t \\
& =\left[M r^{-1}(B) \int_{0}^{1} t x^{*}(t) d t\right] K(\tau, s),
\end{aligned}
$$

Set

$$
f^{*}(u)=\int_{0}^{1} x^{*}(t) u(t) d t, \quad \forall u \in X, t \in[0,1] .
$$

Obviously, $f^{*} \in P^{*} \backslash\{\theta\}$, and by (34), for $u \in X$, we have

$$
\begin{aligned}
f^{*}(B u) & =\int_{0}^{1} x^{*}(t)(B u)(t) d t \\
& =\int_{0}^{1} x^{*}(t) d t \int_{0}^{1} K(t, s) u(s) d s \\
& =\int_{0}^{1} \int_{0}^{1} K(t, s) x^{*}(t) u(s) d t d s \\
& =\int_{0}^{1}\left(\int_{0}^{1} K^{*}(s, t) x^{*}(t) d t\right) u(s) d s \\
& =\int_{0}^{1} r(B) x^{*}(s) u(s) d s=r(B) f^{*}(u) .
\end{aligned}
$$

That is,

$$
B^{*} f^{*}=r(B) f^{*} \text {. }
$$

From (33) and (35), we have

$$
\begin{aligned}
f^{*}(B u) & =r(B) \int_{0}^{1} x^{*}(s) u(s) d s \\
& \geq M \int_{0}^{1} x^{*}(t) t d t \int_{0}^{1} K(\tau, s) u(s) d s \\
& =\left(M \int_{0}^{1} t x *(t) d t\right)(B u)(\tau),
\end{aligned}
$$

$\forall \tau \in[0,1]$

By (37), we have

$$
f^{*}(B u) \geq \delta\|B u\|
$$

where $\delta=M \int_{0}^{1} t x^{*}(t) d t>0$.

Therefore, from (31), (36), and (38), it is easy to know that the linear operator $B$ satisfies $\mathrm{H}$ condition.

Theorem 7. Suppose that $\left(\mathrm{C}_{1}\right),\left(\mathrm{C}_{2}\right)$, and $\left(\mathrm{C}_{3}\right)$ hold. In addition, assume that there exists $\gamma>0$ such that

$$
\liminf _{x \rightarrow+\infty} \frac{\varphi(t, x)}{x} \geq \lambda_{1}+\gamma, \quad \text { uniformly on } t \in[0,1] \text {; }
$$

$\limsup _{x \rightarrow-\infty} \frac{\varphi(t, x)}{x} \leq \lambda_{1}-\gamma, \quad$ uniformly on $t \in[0,1]$.

If $\eta \neq \lambda_{1}, \lambda_{2}, \cdots, \lambda_{n}, \cdots$, where $\lambda_{i}$ is defined by $\left(\mathrm{C}_{2}\right)$, then the boundary value problem (1), (2) has at least one nontrivial solution.

Proof. By $\left(\mathrm{C}_{1}\right)$, we easily know that $A: X \rightarrow X$ is a completely continuous operator, and $B: X \rightarrow X$ is a bounded positive linear completely continuous operator (see [3]). By Lemma 6, we know that the linear operator $B$ satisfies $\mathrm{H}$ condition.

For $x \in X$, let

$$
\begin{aligned}
& x_{+}(t)= \begin{cases}x(t), & x(t) \geq 0, \\
0, & x(t)<0,\end{cases} \\
& x_{-}(t)= \begin{cases}x(t), & x(t) \leq 0, \\
0, & x(t)>0,\end{cases}
\end{aligned}
$$

and then $x(t)=x_{+}(t)+x_{-}(t)$.

By $\varphi(t, 0)=0$, we have

$$
\begin{aligned}
\Phi(x) & =\varphi(t, x(t))=\varphi\left(t, x_{+}(t)+x_{-}(t)\right) \\
& =\varphi\left(t, x_{+}(t)\right)+\varphi\left(t, x_{-}(t)\right) \\
& =\Phi\left(x_{+}\right)+\Phi\left(x_{-}\right) .
\end{aligned}
$$

From (42), we know that $\Phi$ is quasi-additive on lattice.

From (39) and (40), there exists $C>0$ such that

$$
\begin{aligned}
& \frac{\varphi(t, x)}{x} \geq \lambda_{1}+\frac{\gamma}{4}, \quad \forall x \geq C, t \in[0,1], \\
& \frac{\varphi(t, x)}{x} \leq \lambda_{1}-\frac{\gamma}{4}, \quad \forall x \leq-C, t \in[0,1] .
\end{aligned}
$$


By (43) and (44), we have

$$
\begin{aligned}
& \varphi(t, x) \geq\left(\lambda_{1}+\frac{\gamma}{4}\right) x, \quad \forall x \geq C, t \in[0,1], \\
& \varphi(t, x) \geq\left(\lambda_{1}-\frac{\gamma}{4}\right) x, \quad \forall x \leq-C, t \in[0,1] .
\end{aligned}
$$
have

Let $\widetilde{C}=\max _{0 \leq t \leq 1,|x| \leq C}|\varphi(t, x)|$. Then by (45) and (46), we

$$
\begin{aligned}
& \varphi(t, x) \geq\left(\lambda_{1}+\frac{\gamma}{4}\right) x-\widetilde{C}, \quad \forall x \geq 0, t \in[0,1], \\
& \varphi(t, x) \geq\left(\lambda_{1}-\frac{\gamma}{4}\right) x-\widetilde{C}, \quad \forall x \leq 0, t \in[0,1],
\end{aligned}
$$

i.e.,

$$
\begin{aligned}
& \Phi x \geq h_{1} x-\widetilde{C}, \quad \forall x \in P, \\
& \Phi x \geq h_{2} x-\widetilde{C}, \quad \forall x \in(-P),
\end{aligned}
$$

where $h_{1}=\lambda_{1}+\gamma / 4, h_{2}=\lambda_{1}-\gamma / 4$. Obviously, we have

$$
\begin{aligned}
& h_{1}>r^{-1}(B), \\
& h_{2}<r^{-1}(B) .
\end{aligned}
$$

In the following, we prove that $A_{\theta}^{\prime}=\eta B$.

In fact, by $\varphi(t, 0)=0, \forall t \in[0,1]$, we have $A \theta=\theta$. From $\left(\mathrm{C}_{3}\right), \forall \epsilon>0, \exists \delta>0$, when $0<|x|<\delta$, we have

$$
\left|\frac{\varphi(t, x)}{x}-\eta\right|<\epsilon
$$

i.e.,

$$
|\varphi(t, x)-\eta x|<\epsilon|x|, \quad \forall t \in[0,1], 0<|x|<\delta .
$$

So

$$
\|\Phi x-\eta x\| \leq \epsilon\|x\|, \quad \forall\|x\|<\delta .
$$

Therefore, by (52), we have

$$
\begin{aligned}
\|A x-A \theta-\eta B x\| & =\|B(\Phi x-\eta x)\| \\
\leq\|B\| \cdot\|(\Phi x-\eta x)\| \leq \epsilon\|B\| \cdot\|x\|, & \forall\|x\|<\delta .
\end{aligned}
$$

So

$$
\lim _{\|x\| \rightarrow 0} \frac{\|A x-A \theta-\eta B x\|}{\|x\|}=0
$$

i.e.,

$$
A_{\theta}^{\prime}=\eta B
$$

Since $r\left(A_{\theta}^{\prime}\right)=\eta r(B)$, we know that 1 is not the eigenvalue of $A_{\theta}^{\prime}$ by Lemma 6 and $\left(\mathrm{C}_{2}\right)$.

By the above proof, we know that the conditions of Lemma 3 hold. So by Lemma 3, the boundary value problem (1), (2) has at least one nontrivial solution.
Theorem 8. Assume that $\left(\mathrm{C}_{1}\right)-\left(\mathrm{C}_{3}\right),(39)$, and (40) are satisfied. In addition, suppose that $\varphi(t, x) x>0, \forall t \in[0,1], x \neq 0$, and $\lambda_{2 n_{0}}<\eta<\lambda_{2 n_{0}+1}$, where $n_{0}$ is a natural number. Then the boundary value problem (1), (2) has at least one negative solution and one sign-changing solution.

Proof. By (17), for $\forall x \in P \backslash\{\theta\}$, we have

$$
\begin{aligned}
&(B x)(t)= \int_{0}^{1} K(t, s) x(s) d s \\
&= \int_{0}^{1} g(t, s) x(s) d s \\
&+\frac{t \sum_{i=1}^{m-2} \beta_{i} \int_{0}^{1} g\left(\alpha_{i}, s\right) x(s) d s}{1-\sum_{i=1}^{m-2} \beta_{i} \alpha_{i}} \\
& \leq t(1-t) \int_{0}^{1} x(s) d s \\
&+\frac{t \sum_{i=1}^{m-2} \beta_{i}}{1-\sum_{i=1}^{m-2} \beta_{i} \alpha_{i}} \int_{0}^{1} x(s) d s \\
&= {\left.\left[t(1-t)+\frac{t \sum_{i=1}^{m-2} \beta_{i}}{1-\sum_{i=1}^{m-2} \beta_{i} \alpha_{i}}\right]\right]_{0}^{1} x(s) d s . } \\
&(B x)(t)= \int_{0}^{1} g(t, s) x(s) d s \\
&+\frac{t \sum_{i=1}^{m-2} \beta_{i} \int_{0}^{1} g\left(\alpha_{i}, s\right) x(s) d s}{1-\sum_{i=1}^{m-2} \beta_{i} \alpha_{i}} . \\
& 1-\sum_{i=1}^{m-2} \beta_{i} \alpha_{i} \\
&\left(\alpha_{i}, s\right) x(s) d s
\end{aligned}
$$

From (56) and (57), we obtain that

$$
B(P \backslash\{\theta\}) \subset \operatorname{int} P .
$$

Similarly, we know that

$$
B(-P \backslash\{\theta\}) \subset \operatorname{int}(-P) .
$$

Since $\varphi(t, x) x>0, \forall t \in[0,1], x \neq 0$, we have $\varphi(t, x)>$ $0, \forall x>0, t \in[0,1]$, and $\varphi(t, x)<0, \forall x<0, t \in[0,1]$. So we have

$$
\begin{aligned}
& (\Phi x) \in P \backslash\{\theta\}, \quad \forall x \in P \backslash\{\theta\} . \\
& (\Phi x) \in(-P) \backslash\{\theta\}, \quad \forall x \in(-P) \backslash\{\theta\} .
\end{aligned}
$$

By (58)-(61), we have

$$
\begin{gathered}
A(P \backslash\{\theta\}) \subset \operatorname{int} P, \\
A((-P) \backslash\{\theta\}) \subset \operatorname{int}(-P) .
\end{gathered}
$$

Let $\beta$ be the sum of algebraic multiplicities for all the eigenvalues of $A_{\theta}^{\prime}$, lying in the interval $(1, \infty)$. By $(55)$, Lemma 5, and $\lambda_{2 n_{0}}<\eta<\lambda_{2 n_{0}+1}$, we know that

$$
\beta=2 n_{0} \text {. }
$$


By (62) and (63), we know that the conditions (iv) and (v) in Lemma 4 hold. By the proof of Theorem 7, the conditions (i), (ii), and (iii) in Lemma 4 are satisfied. Therefore, by Lemma 4, the boundary value problem (1), (2) has at least one negative solution and one sign-changing solution.

\section{Examples}

We consider second-order four-point boundary value problem

$$
\begin{aligned}
-x^{\prime \prime}(t) & =\varphi(t, x(t)), \quad 0 \leq t \leq 1, \\
x(0) & =0, \\
x(1) & =\frac{1}{3} x\left(\frac{1}{3}\right)+\frac{1}{2} x\left(\frac{1}{2}\right) .
\end{aligned}
$$

By simple calculations, $\lambda_{1} \approx 5.602, \lambda_{2} \approx 42.32, \lambda_{3} \approx$ 99.97, and $\lambda_{4} \approx 148.87$ are solutions of the equation

$$
\sin \sqrt{x}=\frac{1}{3} \sin \frac{\sqrt{x}}{3}+\frac{1}{2} \sin \frac{\sqrt{x}}{2} .
$$

Example 1. Choose

$$
\begin{aligned}
& \varphi(t, x) \\
& = \begin{cases}8 x+(t-1) \sqrt{x}, & t \in[0,1], x \in[4,+\infty), \\
\frac{30+5 t}{3}(x-1)-3 t, & t \in[0,1], x \in(1,4), \\
3 x-3(1+t) x^{2}, & t \in[0,1], x \in[-1,1], \\
\frac{8-t}{7}(x+1)-(6+3 t), & t \in[0,1], x \in(-8,-1), \\
2 x+(t-1) \sqrt[3]{x}, & t \in[0,1], x \in(-\infty,-8] .\end{cases}
\end{aligned}
$$

By (66), it is easy to know that $\varphi:[0,1] \times(-\infty,+\infty) \longrightarrow$ $(-\infty,+\infty)$ is continuous, and $\varphi(t, 0)=0, \forall t \in[0,1]$. By calculation, $\eta=3<\lambda_{1}$. We can choose $\gamma=2$. Then we have

$$
\begin{aligned}
& \liminf _{x \rightarrow+\infty} \frac{\varphi(t, x)}{x}=8 \geq \lambda_{1}+\gamma, \\
& \limsup _{x \rightarrow-\infty} \frac{\varphi(t, x)}{x}=2 \leq \lambda_{1}-\gamma .
\end{aligned}
$$

So by Theorem 7 , the boundary value problem (64) has at least one nontrivial solution.

\section{Example 2. Choose}

$$
\begin{aligned}
& \varphi(t, x) \\
& = \begin{cases}10 x+(1-t) \sqrt[3]{x}, & t \in[0,1], x \in[8,+\infty), \\
\frac{31-3 t}{7}(x-1)+(51+t), & t \in[0,1], x \in(1,8), \\
50 x+(1+t) x^{5 / 3}, & t \in[0,1], x \in[-1,1], \\
\frac{-21-4 t}{26}(x+1)-(51+t), & t \in[0,1], x \in(-27,-1), \\
x+(1-t) \sqrt[3]{x}, & t \in[0,1], x \in(-\infty,-27] .\end{cases}
\end{aligned}
$$

By (68), we know that $\varphi:[0,1] \times(-\infty,+\infty) \longrightarrow$ $(-\infty,+\infty)$ is continuous, $\varphi(t, 0)=0, \forall t \in[0,1]$, and $\varphi(t, x) x>0, \forall t \in[0,1], x \neq 0$. By calculation, $\lambda_{2} \leq \eta=$ $50<\lambda_{3}$. We can choose $\gamma=4$. Then we have

$$
\begin{aligned}
& \liminf _{x \rightarrow+\infty} \frac{\varphi(t, x)}{x}=10 \geq \lambda_{1}+\gamma, \\
& \limsup _{x \rightarrow-\infty} \frac{\varphi(t, x)}{x}=1 \leq \lambda_{1}-\gamma .
\end{aligned}
$$

So by Theorem 8 , the boundary value problem (64) has at least one negative solution and one sign-changing solution.

Example 3. Choose

$$
\begin{aligned}
& \varphi(t, x) \\
& = \begin{cases}\frac{45}{32} x^{2}+(1-t) \sqrt[3]{x}, & t \in[0,1], x \in[8,+\infty), \\
\frac{31-3 t}{7}(x-1)+(61+t), & t \in[0,1], x \in(1,8), \\
60 x+(1+t) x^{5 / 3}, & t \in[0,1], x \in[-1,1], \\
\frac{-31-4 t}{26}(x+1)-(61+t), & t \in[0,1], x \in(-27,-1), \\
x+(1-t) \sqrt[3]{x}, & t \in[0,1], x \in(-\infty,-27] .\end{cases}
\end{aligned}
$$

By (70), we know that $\varphi:[0,1] \times(-\infty,+\infty) \longrightarrow$ $(-\infty,+\infty)$ is continuous, $\varphi(t, 0)=0, \forall t \in[0,1]$, and $\varphi(t, x) x>0, \forall t \in[0,1], x \neq 0$. By calculation, $\lambda_{2} \leq \eta=$ $60<\lambda_{3}$. We can choose $\gamma=3$. Then we have

$$
\begin{aligned}
& \liminf _{x \rightarrow+\infty} \frac{\varphi(t, x)}{x}=+\infty \geq \lambda_{1}+\gamma, \\
& \limsup _{x \rightarrow-\infty} \frac{\varphi(t, x)}{x}=1 \leq \lambda_{1}-\gamma .
\end{aligned}
$$

So by Theorem 8 , the boundary value problem (64) has at least one negative solution and one sign-changing solution.

\section{Data Availability}

The date underlying the findings of our manuscript can be obtained by simple calculations. We did not quote other data.

\section{Conflicts of Interest}

The authors declare that they have no conflicts of interest regarding the publication of this paper.

\section{Acknowledgments}

The project is supported by the Youth Science Foundation of China (11801322), the National Natural Science Foundation of China (11571207), and Shandong Natural Science Foundation (ZR2018MA011).

\section{References}

[1] C. Gupta, "Solvability of a three-point nonlinear boundary value problem for a second order ordinary differential equation," Journal of Mathematical Analysis and Applications, vol. 168 , no. 2, pp. 540-551, 1992. 
[2] X. Xu, "Multiple sign-changing solutions for some m-point boundary value problems," Electronic Journal of Differential Equations, vol. 2004, no. 89, pp. 1-14, 2004.

[3] G. Zhang and J. Sun, "Existence of positive solutions for singular second order m-point boundary value problems," Acta Mathematicae Applicatae Sinica, vol. 21, no. 4, pp. 655-664, 2004.

[4] K. Zhang and X. Xie, "Existence of sign-changing solutions for some asymptotically linear three-point boundary value problems," Nonlinear Analysis: Theory, Methods \& Applications, vol. 70, no. 7, pp. 2796-2805, 2009.

[5] J. Jiang, L. Liu, and Y. Wu, "Symmetric positive solutions to singular system with multi-point coupled boundary conditions," Applied Mathematics and Computation, vol. 220, no. 4, pp. 536548, 2013

[6] H. Li, "Existence of nontrivial solutions for unilaterally asymptotically linear three-point boundary value problems," Abstract and Applied Analysis, vol. 2014, Article ID 263042, 7 pages, 2014.

[7] Y. Cui, L. Liu, and X. Zhang, "Uniqueness and existence of positive solutions for singular differential systems with coupled integral boundary value problems," Abstract and Applied Analysis, vol. 2013, Article ID 340487, 9 pages, 2013.

[8] Y. Zou, L. Liu, and Y. Cui, “The existence of solutions for fourpoint coupled boundary value problems of fractional differential equations at resonance," Abstract and Applied Analysis, vol. 2014, Article ID 314083, 8 pages, 2014.

[9] Z. Bai, "Eigenvalue intervals for a class of fractional boundary value problem," Computers \& Mathematics with Applications, vol. 64, no. 10, pp. 3253-3257, 2012.

[10] Y. Cui, "Uniqueness of solution for boundary value problems for fractional differential equations," Applied Mathematics Letters, vol. 51, pp. 48-54, 2016.

[11] X. Dong, Z. Bai, and S. Zhang, "Positive solutions to boundary value problems of $p$-Laplacian with fractional derivative," Boundary Value Problems, vol. 2017, article no. 5, 15 pages, 2017.

[12] Z. Bai, S. Zhang, S. Sun, and C. Yin, "Monotone iterative method for a class of fractional differential equations," Electronic Journal of Differential Equations, vol. 2016, no. 6, pp. 1-8, 2016.

[13] Y. Cui and Y. Zou, "Existence results and the monotone iterative technique for nonlinear fractional differential systems with coupled four-point boundary value problems," Abstract and Applied Analysis, vol. 2014, Article ID 242591, 6 pages, 2014.

[14] H. Li and J. Zhang, "Global structure of positive solutions for some second-order multipoint boundary value problems," Journal of Function Spaces, vol. 2017, Article ID 1014250, 6 pages, 2017.

[15] L. Guo, L. Liu, and Y. Wu, "Existence of positive solutions for singular higher-order fractional differential equations with infinite-point boundary conditions," Boundary Value Problems, vol. 2016, article no. 114, 22 pages, 2016.

[16] R. Pu, X. Zhang, Y. Cui, P. Li, and W. Wang, "Positive solutions for singular semipositone fractional differential equation subject to multipoint boundary conditions," Journal of Function Spaces, vol. 2017, Article ID 5892616, 7 pages, 2017.

[17] H. Li, "Existence of nontrivial solutions for superlinear threepoint boundary value problems," Acta Mathematicae Applicatae Sinica, vol. 33, no. 4, pp. 1043-1052, 2017.

[18] M. Zuo, X. Hao, L. Liu, and Y. Cui, "Existence results for impulsive fractional integro-differential equation of mixed type with constant coefficient and antiperiodic boundary conditions," Boundary Value Problems, vol. 2017, article no. 161, 15 pages, 2017.
[19] Y. Cui, W. Ma, Q. Sun, and X. Su, "New uniqueness results for boundary value problem of fractional differential equation," Nonlinear Analysis: Modelling and Control, vol. 23, no. 1, pp. 3139, 2018.

[20] Z. Bai, X. Dong, and C. Yin, "Existence results for impulsive nonlinear fractional differential equation with mixed boundary conditions," Boundary Value Problems, vol. 2016, article no. 63, 11 pages, 2016.

[21] Q. Sun, H. Ji, and Y. Cui, "Positive solutions for boundary value problems of fractional differential equation with integral boundary conditions," Journal of Function Spaces, vol. 2018, Article ID 6461930, 6 pages, 2018.

[22] Y. Cui and Y. Zou, "An existence and uniqueness theorem for a second order nonlinear system with coupled integral boundary value conditions," Applied Mathematics and Computation, vol. 256, pp. 438-444, 2015.

[23] J. Zhang, G. Zhang, and H. Li, "Positive solutions of secondorder problem with dependence on derivative in nonlinearity under Stieltjes integral boundary condition," Electronic Journal of Qualitative Theory of Differential Equations, no. 4, pp. 1-13, 2018.

[24] X. Qiu, J. Xu, D. O’Regan, and Y. Cui, "Positive solutions for a system of nonlinear semipositone boundary value problems with Riemann-Liouville fractional derivatives," Journal of Function Spaces, vol. 2018, Article ID 7351653, 10 pages, 2018.

[25] X. Zhang and Q. Zhong, "Uniqueness of solution for higherorder fractional differential equations with conjugate type integral conditions," Fractional Calculus and Applied Analysis, vol. 20, no. 6, pp. 1471-1484, 2017.

[26] J. Wu, X. Zhang, L. Liu, Y. Wu, and Y. Cui, “The convergence analysis and error estimation for unique solution of a $\mathrm{p}$ Laplacian fractional differential equation with singular decreasing nonlinearity," Boundary Value Problems, vol. 2018, article no. 82, 15 pages, 2018.

[27] X. Zhang, L. Liu, Y. Wu, and Y. Zou, "Existence and uniqueness of solutions for systems of fractional differential equations with Riemann-Stieltjes integral boundary condition," Advances in Difference Equations, vol. 2018, article no. 204, 15 pages, 2018.

[28] J. Sun and X. Liu, "Computation of topological degree for nonlinear operators and applications," Nonlinear Analysis. Theory, Methods \& Applications. An International Multidisciplinary Journal, vol. 69, no. 11, pp. 4121-4130, 2008.

[29] X. Liu and J. Sun, "Computation of topological degree of unilaterally asymptotically linear operators and its applications," Nonlinear Analysis. Theory, Methods \& Applications. An International Multidisciplinary Journal, vol. 71, no. 1-2, pp. 96106, 2009.

[30] J. Sun and X. Liu, "Computation of topological degree in ordered Banach spaces with lattice structure and its application to superlinear differential equations," Journal of Mathematical Analysis and Applications, vol. 348, no. 2, pp. 927-937, 2008.

[31] J. Sun, Nonlinear Functional Analysis and Applications, Science Press, Beijing, China, 2007.

[32] Y. Cui, "Computation of topological degree in ordered banach spaces with lattice structure and applications," Applications of Mathematics, vol. 58, no. 6, pp. 689-702, 2013.

[33] J. Sun and Y. Cui, "Fixed point theorems for a class of nonlinear operators in Riesz spaces," Fixed Point Theory and Applications, vol. 14, no. 1, pp. 185-192, 2013. 
[34] Y. Cui and J. Sun, "Fixed point theorems for a class of nonlinear operators in Hilbert spaces with lattice structure and application," Fixed Point Theory and Applications, vol. 2013, article no. 345, 9 pages, 2013.

[35] H. Li and F. Sun, "Existence of solutions for integral boundary value problems of second-order ordinary differential equations," Boundary Value Problems, vol. 2012, article no. 147, 7 pages, 2012.

[36] H. Xu, "Existence of nontrivial solutions and sign-changing solutions for nonlinear dynamic equations on time scales," Discrete Dynamics in Nature and Society, vol. 2011, Article ID 604170, 22 pages, 2011.

[37] H. Lu, L. Sun, and J. Sun, "Existence of positive solutions to a non-positive elastic beam equation with both ends fixed," Boundary Value Problems, vol. 2012, article no. 56, 10 pages, 2012.

[38] K. Deimling, Nonlinear Functional Analysis, Springer, Berlin, Germany, 1985.

[39] D. Guo and V. Lakshmikantham, Nonlinear Problems in Abstract Cones, Academic Press, San Diego, Calif, USA, 1988.

[40] W. Luxemburg and A. Zaanen, Riesz Spaces, vol. I, NorthHolland Publishing Company, London, UK, 1971.

[41] J. Sun, "Nontrivial solutions of superlinear Hammerstein integral equations and their applications," Chinese Annals of Mathematics, vol. 7, no. 5, pp. 528-535, 1986 (Chinese). 


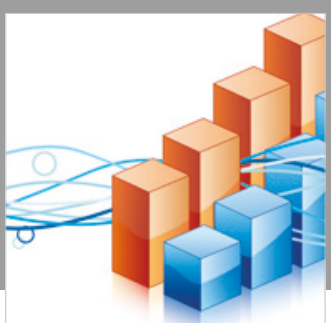

Advances in

Operations Research

\section{-n-m}
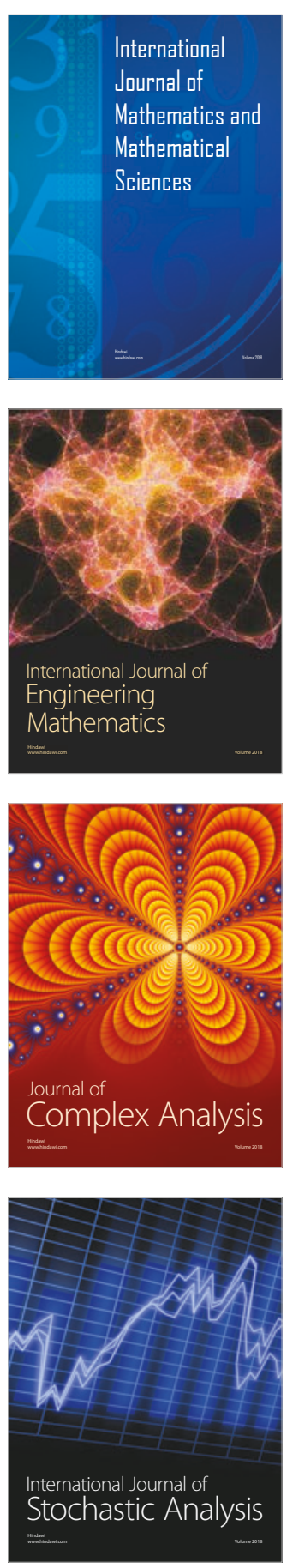
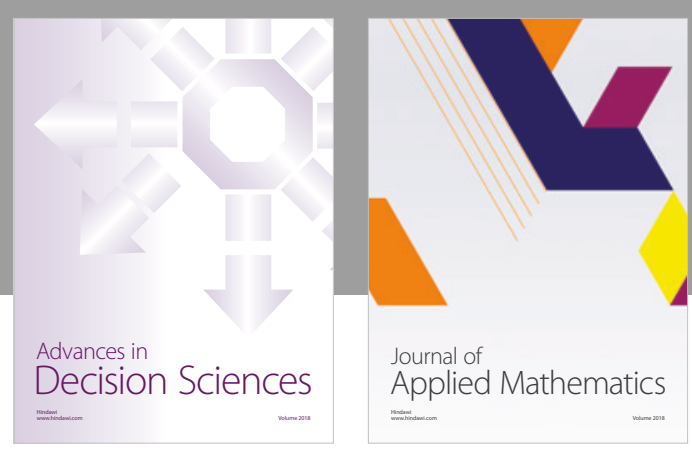

Journal of

Applied Mathematics
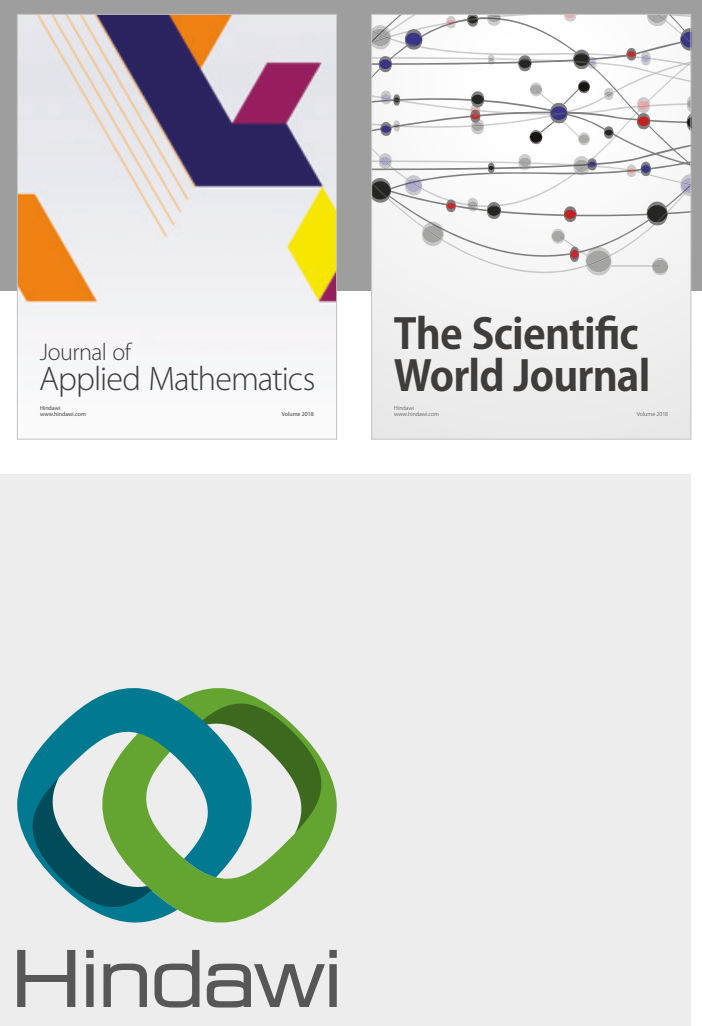

Submit your manuscripts at

www.hindawi.com

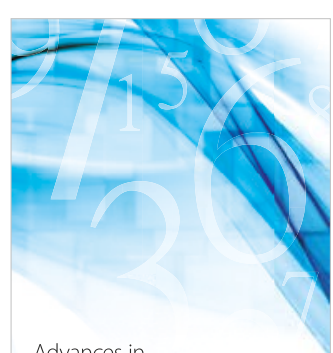

Advances in
Numerical Analysis
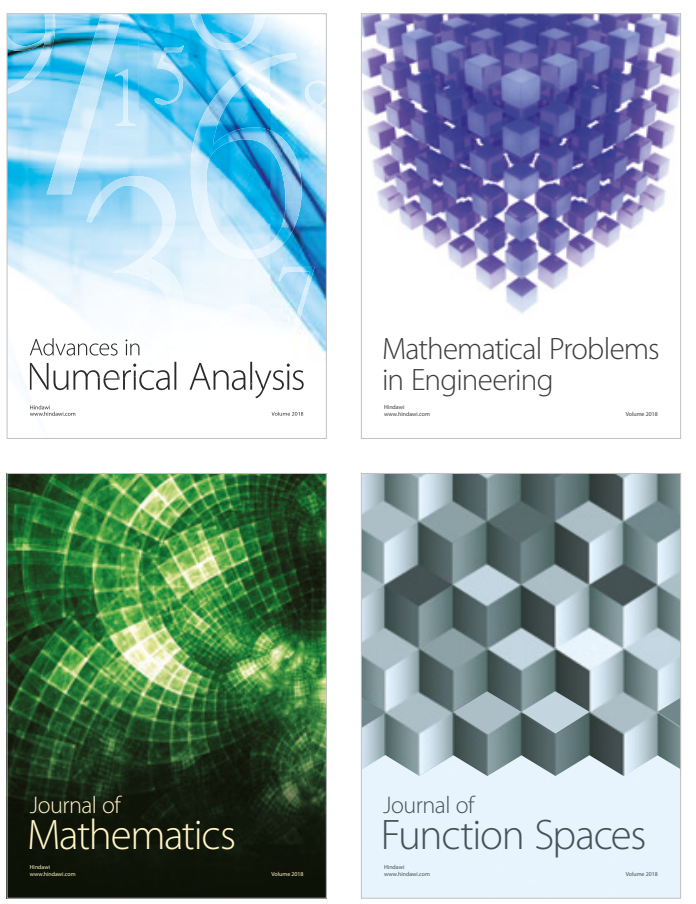

Mathematical Problems in Engineering

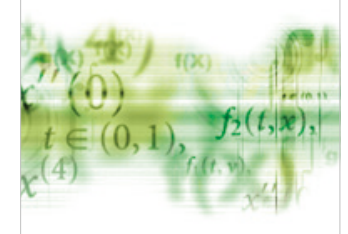

International Journal of

Differential Equations

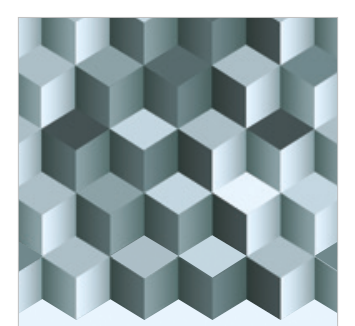

Journal of

Function Spaces

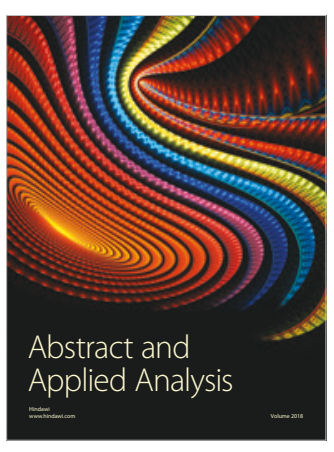

The Scientific

World Journal

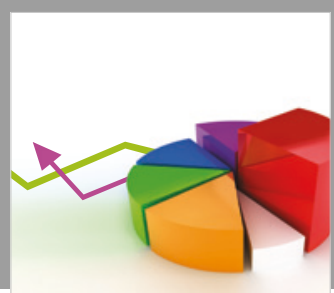

Journal of

Probability and Statistics
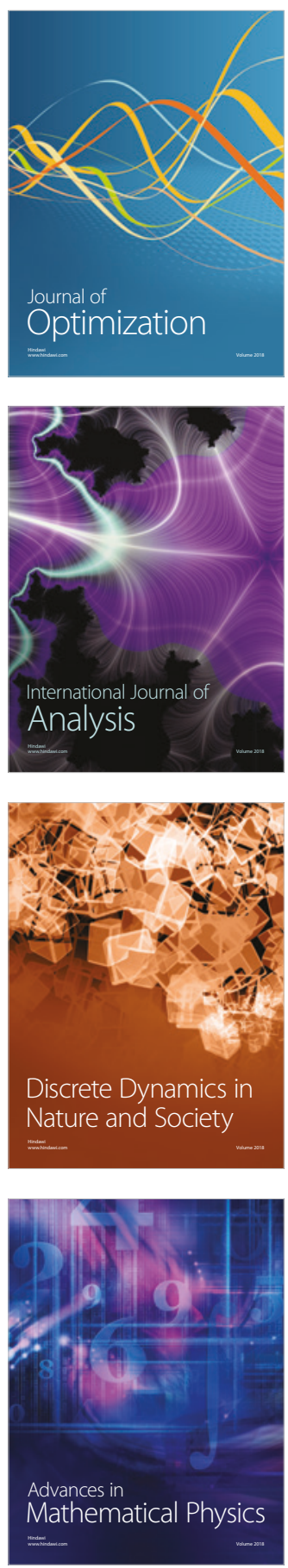